\title{
Retos de la Organización Mundial del Comercio como consecuencia de la Undécima Conferencia Ministerial en Buenos Aires
}

\author{
Humberto Zúñiga Schroder*
}

\section{RESUMEN}

La Organización Mundial del Comercio es una institución que, a la fecha, cuenta con más de 20 ańos de existencia. La última conferencia ministerial, celebrada en Buenos Aires en diciembre del año 2017, ha planteado algunos retos que deben ser abordados por todos sus miembros, no solo con el objetivo de fortalecer el sistema multilateral de comercio, sino también para llevar a buen término la Ronda de Doha, iniciada en el ańo 2001. El objeto del presente trabajo es analizar las más importantes decisiones ministeriales alcanzadas en Buenos Aires, y dar una mirada breve a la naturaleza de los problemas que sustentaron la emisión de las mismas.

Palabras clave: Organización Mundial del Comercio, subvenciones, pesca, propiedad intelectual, comercio electrónico.

Challenges of the World Trade Organization as a consequence of the eleventh ministerial conference held in Buenos Aires

\section{Abstract}

The World Trade Organization is an international body that, to date, has more than 20 years of existence. The last ministerial conference, held in Buenos Aires in December of 2017, has posed some challenges that must be addressed by all its members, not only to strengthen the multilateral trading system, but also to achieve a successful outcome of the Doha Round, which started in 2001. The purpose of this paper is to analyze the most important ministerial decisions reached in Buenos Aires, and to give a brief insight into the nature of the problems that were addressed in such decisions.

Keywords: World Trade Organization, subsidies, fisheries, intellectual property, e-commerce.

\footnotetext{
* Vicepresidente del Comité Permanente de Normalización del INACAL y miembro de la Secretaría Técnica de la Comisión de Dumping, Subsidios y Eliminación de Barreras Comerciales No Arancelarias del Indecopi. Profesor de Derecho en la Pontificia Universidad Católica del Perú y en la Universidad de Piura. Doctor en Derecho (Ph.D.) por la Universidad de Edimburgo; magíster en Derecho del Comercio Internacional y Europeo (LL.M.) por la Universidad de Ámsterdam, y abogado por la Pontificia Universidad Católica del Perú. Correo electrónico: hzschroder@gmail.com.
} 


\section{Introducción}

Desde su concepción, la Organización Mundial del Comercio (OMC) ha tenido una influencia innegable en el escenario mundial, gracias a la implementación de un conjunto de normas destinadas a dar claridad, predictibilidad y seguridad a sus miembros en sus intercambios y transacciones comerciales. No obstante, es claro también que la OMC cuenta con incontables detractores, mucho de los cuales han criticado la posición desventajosa de diversos países en desarrollo al interior de este organismo, falta de transparencia, e irregularidades en los procesos de negociación de acuerdos. Pese a ello, lo cierto es que, a la fecha, la OMC cuenta con 164 miembros, y 22 se encuentran en proceso de adhesión; es decir, el llamado «sistema multilateral de comercio» ha demostrado ser, en términos generales, efectivo, pese a los retos que actualmente enfrenta.

Uno de los retos actuales que enfrenta la $\mathrm{OMC}$ es el poder llevar a buen término la denominada Ronda de Doha, iniciada en el año 2001. Esta ronda —la cual ha probado ser, a la fecha, la más extensa a nivel de la OMC e incluso dentro del GATT $^{1}$ - busca lograr una reforma integral del sistema de comercio internacional, mediante la adopción de decisiones en temas tan disímiles como lo pueden ser la agricultura, acceso a los mercados para productos no agrícolas (AMNA), facilitación del comercio, normas, medio ambiente y propiedad intelectual, entre otros. A la fecha, las negociaciones no han resultado sencillas — particularmente, en ciertos ámbitos como por ejemplo la agricultura, donde ha sido ciertamente difícil alcanzar consenso entre los participantes - siendo que, en la última Conferencia Ministerial del año 2017, los miembros buscaron avanzar con el mandato impuesto en esta ronda, con el propósito último de definir las nuevas reglas generales destinadas a facilitar el intercambio global. En virtud de ello, el presente trabajo estará dedicado a analizar las principales decisiones ministeriales alcanzadas en esta última conferencia ministerial; ello, a efectos de poder entender cuáles son los principales retos que este organismo deberá enfrentar, y los temas que serán objeto de negociación y eventual acuerdo por parte de sus miembros, en los próximos años.

\section{Sobre las conferencias ministeriales de la OMC}

La OMC es un organismo internacional, creado en 1995, y que tiene entre sus funciones resolver las diferencias comerciales entre sus distintos miembros;

\footnotetext{
1 Acuerdo General sobre Aranceles Aduaneros y Comercio. Si bien el GATT fue originalmente concebido como un acuerdo, en la práctica operó como organización encargada de supervisar el sistema multilateral de comercio, desde el ańo 1947, hasta el establecimiento de la OMC, el 1 de enero de 1995.
} 
administrar los acuerdos que conforman el sistema multilateral de comercio, y supervisar las políticas comerciales nacionales de sus integrantes. Además, constituye el foro para negociaciones comerciales y coopera con otras organizaciones internacionales $^{2}$. Actualmente, dicha institución se encuentra conformada por 164 miembros, entre ellos el Perú (OMC, s.f. a).

El órgano máximo para la adopción de decisiones al interior de la $\mathrm{OMC}$ es la Conferencia Ministerial, conformada por representantes de todos los Miembros, y que habitualmente se reúne cada dos años, teniendo potestad para adoptar decisiones sobre cualquier asunto incluido dentro del ámbito de aplicación de los Acuerdos Comerciales Multilaterales de dicha institución (OMC, s.f. b). Además, según lo prescrito en el Acuerdo por el que se establece la Organización Mundial del Comercio, la Conferencia Ministerial tiene capacidad para adoptar interpretaciones de cualquier acuerdo multilateral anexo a dicho instrumento, eximir a miembros de la OMC de sus obligaciones, adoptar enmiendas, y decidir tanto la adhesión de nuevos miembros como el nombramiento del Director General de la OMC 3 .

Desde la conformación de la OMC se han llevado a cabo 11 conferencias ministeriales, siendo que la última de ellas tuvo lugar en Buenos Aires, Argentina, del $10 \mathrm{al}$ 13 de diciembre del $2017^{4}$. Esta conferencia concluyó con la adopción de diversas decisiones ministeriales, las cuales definirán la política de acción a ser adoptada por este organismo a futuro; sin perjuicio, claro, de las demás negociaciones que vienen realizándose en otros ámbitos, al amparo del mandato impuesto en Doha ${ }^{5}$.

Si bien no fue posible alcanzar un acuerdo con relación a varias cuestiones importantes; por ejemplo, asuntos relacionados con la agricultura o la constitución de existencias públicas con fines de seguridad alimentaria (OMC, s.f. c), los miembros pudieron adoptar decisiones respecto de los siguientes cinco temas: comercio electrónico, subvenciones a la pesca, reclamaciones en el ámbito de los ADPIC, programa

\footnotetext{
2 De manera adicional, la OMC tiene entre sus funciones brindar asistencia técnica y cursos de formación para los países en desarrollo. Véase el artículo III del Acuerdo por el que se establece la Organización Mundial del Comercio. 3 Véase, respectivamente, los artículos IX:2, IX:3, X, XII y VI:2 del Acuerdo por el que se establece la Organización Mundial del Comercio.

4 Estas conferencias han tenido lugar en: Singapur (1996), Ginebra (1998), Seattle (1999), Doha (2001), Cancún (2003), Hong Kong (2005), Ginebra (2009 y 2011), Bali (2013), Nairobi (2015) y Buenos Aires (2017).

5 Esta conferencia contó con la participación de representantes de los 164 miembros de la OMC. Es importante mencionar que, durante esta conferencia, tuvieron lugar acontecimientos destacables, como la publicación de la Declaración de Buenos Aires sobre las mujeres y el comercio, y la puesta en marcha de la iniciativa de promoción del comercio electrónico ( Enabling E-commerce»), entre otros. Además, tres grupos de proponentes anunciaron nuevas iniciativas para hacer avanzar las negociaciones en la OMC sobre cuestiones relacionadas con el comercio electrónico, la facilitación de las inversiones y las microempresas y pequeñas y medianas empresas (MIPYME). Véase la página web de la OMC: https://www.wto.org/spanish/thewto_s/minist_s/mc11_s/mc11_s.htm
} 
de trabajo sobre las pequeñas economías y la creación de un Grupo de Trabajo sobre la adhesión de Sudán del Sur.

A efectos del presente trabajo, únicamente las decisiones adoptadas sobre los tres primeros temas serán analizadas en secciones subsiguientes; ello, a efectos de dilucidar su importancia y relevancia actual dentro del régimen comercial internacional, y para efectos de definir qué áreas en concreto serán materia de negociación, en las próximas conferencias ministeriales. Nótese que los dos últimos temas reseñados en el párrafo anterior (programa de trabajo sobre las pequeñas economías y la creación de un Grupo de Trabajo sobre la adhesión de Sudán del Sur) no serán materia de análisis, al no estar directamente relacionados con cuestiones sustanciales de alguno de los acuerdos incluidos dentro del Anexo 1 del Acuerdo por el que se establece la Organización Mundial del Comercio.

\section{Programa de trabajo sobre comercio electrónico}

La primera decisión ministerial a ser analizada tiene que ver con el programa de trabajo sobre comercio electrónico. Nótese que en virtud de esta decisión, los miembros acordaron «continuar la labor realizada en el marco del Programa de Trabajo sobre el Comercio Electrónico» desde el último período de sesiones; y, además, mantener hasta el 2019 (fecha en la cual se llevaría a cabo la próxima conferencia ministerial) «la práctica actual de no imponer derechos de aduana a las transmisiones electrónicas» ${ }^{6}$. Cabe destacar además que el programa de trabajo antes mencionado data del año 1998; es decir, fue adoptado pocos años después de establecida la OMC.

En términos generales, el comercio electrónico (o e-commerce por su denominación en inglés) puede ser definido como la compra y venta de productos o servicios a través de internet, $\mathrm{u}$ otras redes informáticas, a través de la utilización de medios electrónicos de pago, tales como tarjetas de crédito. Similar definición ha sido recogida también por la OMC, la cual entiende por comercio electrónico toda «[...] producción, distribución, comercialización, venta o entrega de bienes y servicios por medios electrónicos» (OMC, s.f. d, párr. 1.3). Además, dicho organismo ha especificado las siguientes tres fases principales que comprende una transacción llevada a cabo por medios electrónicos:

Una transacción comercial puede dividirse en tres fases principales: la fase de publicidad y búsqueda, la fase de pedido y pago y la fase de entrega. Cualquiera de estas fases o todas ellas pueden realizarse electrónicamente y, por lo tanto, quedar incluidas en el concepto de "comercio electrónico. (OMC, s.f. e, párr. 3)

\footnotetext{
6 El texto completo de esta decisión se encuentra disponible en el siguiente documento OMC: WT/L/1032.
} 
La importancia del comercio electrónico, dentro del régimen multilateral de comercio, fue ya reconocida desde la Segunda Conferencia Ministerial —celebrada en mayo de 1998 - en la cual los ministros adoptaron la Declaración sobre el Comercio Electrónico Mundial. De acuerdo con Mitchell, la ventaja de ubicar el comercio electrónico en el marco del régimen de la OMC radica en el hecho de que el sistema multilateral de comercio proporciona un conjunto de reglas transparentes, predecibles y exigibles, las cuales están basadas en los principios de no discriminación ${ }^{7}$ y transparencia, y que son de indiscutible relevancia no solo para el comercio electrónico en sí, sino también para otras formas de comercio internacional (Mitchell, 2001, p. 688).

Ahora bien, en la declaración de 1998, se encomendó al Consejo General el establecimiento de un programa de trabajo amplio destinado a examinar las cuestiones relacionadas con el comercio electrónico mundial que pudiesen afectar el comercio; y, además, los Miembros acordaron mantener su práctica de no imponer derechos de aduanas a las transmisiones electrónicas (es decir, que aplicarían una «moratoria» respecto de la imposición de estos derechos) (OMC, s.f. f). Es importante mencionar que, en el Programa de Trabajo antes citado, los Miembros decidieron encomendar a los tres consejos (Comercio de Mercancías, Servicios, y Consejo de los ADPIC) examinar el trato del comercio electrónico en el marco jurídico del GATT 1994, del Acuerdo General sobre el Comercio de Servicios (AGCS) y del Acuerdo sobre los Aspectos de los Derechos de Propiedad Intelectual relacionados con el Comercio (ADPIC) y, además, encomendaron al Comité de Comercio y Desarrollo que examine las consecuencias del comercio electrónico para el desarrollo (OMC, s.f. d).

La labor del Consejo General no se vio agotada en la elaboración de este programa de trabajo y en los sendos reportes remitidos por los órganos antes citados: a partir de mayo del 2001 en adelante, dicho órgano ha llevado a cabo diversos debates destinados a examinar las cuestiones transversales comprendidas en el Programa de Trabajo referido anteriormente. El primero de esos debates tuvo lugar el 15 de junio de 2001, el cual culminó con la elaboración de un documento de trabajo que resume las cuestiones planteadas por la Secretaría (OMC, s.f. g) ${ }^{8}$.

a. Clasificación del contenido de ciertas transmisiones electrónicas: La mayoría de las delegaciones destacaron la importancia de la clasificación como cuestión

\footnotetext{
Cláusula de nación más favorecida y trato nacional.

Nótese que, de manera adicional, se han llevado a cabo diversas reuniones al interior del Consejo General en las cuales se ha debatido este tema, siendo que anualmente, el presidente ha cumplido con elaborar sendos informes en los cuales describe la problemática e inquietudes de los miembros participantes.
} 
intersectorial. Asimismo, varias delegaciones convinieron en que la mayoría de los productos que se transmitían electrónicamente eran de hecho servicios?.

b. Cuestiones relacionadas con el desarrollo: Todas las delegaciones convinieron en que el desarrollo era una cuestión fundamental en el debate sobre el comercio electrónico. Asimismo, varias delegaciones instaron al Comité de Comercio y Desarrollo (CCD) que siguiera ocupándose de canalizar la información sobre las experiencias y mejores prácticas de los países en lo que respecta al comercio electrónico, a través de la organización de seminarios abiertos a la participación de expertos nacionales.

c. Consecuencias fiscales del comercio electrónico, relación (y posible efecto de sustitución) entre comercio electrónico y formas tradicionales de comercio, imposición de derechos de aduana a las transmisiones electrónicas: Varias delegaciones coincidieron en que la cuestión de las consecuencias fiscales del comercio electrónico justificaba un examen más detallado. Asimismo, hicieron notar que se debería vigilar cuidadosamente la relación entre el comercio electrónico y las formas tradicionales de comercio, ello con el fin de evitar cualquier posible desventaja a corto plazo para los países en desarrollo.

Como puede apreciarse, no solo del análisis del documento antes mencionado, sino también de la opinión de importantes académicos en esta esfera (entre ellos, Panagariya y Mitchell ${ }^{10}$, la prestación electrónica de servicios entra dentro del alcance del AGCS, y en principio, todas las disposiciones del AGCS serían aplicables al comercio de servicios a través de medios electrónicos.

Lo anteriormente mencionado obliga a un breve análisis del alcance del AGCS. La definición del comercio de servicios en el marco del AGCS presenta cuatro modalidades en virtud de la presencia territorial del proveedor y del consumidor en el momento de la transacción. En ese contexto, según el párrafo 2 del artículo I, el AGCS comprende los siguientes tipos o «modos» de servicios (OMC, s.f. h):

a. Modo 1 - Comercio transfronterizo: del territorio de un Miembro al territorio de cualquier otro Miembro;

b. Modo 2 - Consumo en el extranjero: en el territorio de un Miembro a un consumidor de servicios de cualquier otro Miembro;

c. Modo 3 - Presencia comercial: por un proveedor de servicios de un Miembro mediante la presencia comercial en el territorio de cualquier otro Miembro; $y$,

\footnotetext{
9 Sin perjuicio de ello, también destacaron la falta de claridad sobre la cuestión de la clasificación en el marco del GATT o del AGCS de ciertos productos que se pueden entregar tanto en forma electrónica como en soportes físicos. 10 Panagariya, 2000, p. 961: «In my judgment, on balance, it makes more sense to define all electronic transmissions as services». Véase también: Mitchell, 2001, p. 688.
} 
d. $\quad$ Modo 4 - Presencia de personas físicas: por un proveedor de servicios de un Miembro mediante la presencia de personas físicas de un Miembro en el territorio de cualquier otro Miembro.

La existencia de estas 4 modalidades de servicios, tal como se encuentra recogida en el texto actual del AGCS, puede acarrear problemas en la clasificación de las transacciones llevadas a cabo por medios electrónicos, en particular en lo concerniente a los módulos 1 y 2. Así, la OMC ha señalado:

[...] como resultado de los progresos tecnológicos, el modo 1 ha adquirido cada vez más importancia. Varios servicios cuyo suministro requería antańo la proximidad física entre el consumidor y el proveedor hoy pueden ser objeto de comercio por medio de Internet u otras redes. Entre ellos figuran, por ejemplo, la banca electrónica, los servicios de informática y tecnología de la información [...] Aunque el suministro transfronterizo (modo 1) sea el más pertinente, en las transacciones de servicios mediante Internet también puede intervenir el consumo en el extranjero (modo 2). No está claro de qué manera pueden aplicarse los modos de suministro del AGCS a la prestación electrónica y, en general, cómo distinguir en ella los modos de suministro 1 y 2 [...]. Por eso los compromisos con respecto a ambos modos pueden ser aplicables. (OMC, s.f. i) (cursiva agregada).

Un problema adicional a la clasificación en el marco del AGCS, radica en el acceso de los diversos países en desarrollo a los innumerables beneficios derivados del comercio electrónico. Como bien menciona Panagariya, la mayor parte del comercio electrónico se encuentra distribuido de manera desigual, entre países desarrollados y países en desarrollo, siendo que en ciertas regiones (por ejemplo, África) la infraestructura en telecomunicaciones se encuentra tan poco desarrollada que la posibilidad, para los habitantes de estas zonas, de poder beneficiarse de estas actividades tomará, ciertamente, tiempo y esfuerzo (Panagariya, 2000, p. 969). Si bien la creciente adhesión al Acuerdo sobre Tecnología de la Información ${ }^{11}$ —el cual, a la fecha, cuenta con 81 miembros - representa un paso adelante en la mejora de esta infraestructura (al exigir a cada participante la eliminación y consolidación a un nivel nulo los derechos de aduana aplicados a diversos productos especificados en el Acuerdo), existe todavía un largo camino por recorrer para romper esta brecha.

En suma; queda claro que los miembros de la OMC han reparado en la necesidad de regular, de una u otra forma, esta figura, al interior del sistema multilateral de

\footnotetext{
11 El Acuerdo sobre Tecnología de la Información fue adoptado en 1996, y abarca un gran número de productos de alta tecnología, incluidos computadoras, equipos de telecomunicaciones, semiconductores, equipos de fabricación y prueba de semiconductores, soportes lógicos, instrumentos científicos, y casi todas las partes y accesorios de estos productos.
} 
comercio, particularmente al interior del $\mathrm{AGCS}^{12}$. No obstante, lo cierto es que los 164 miembros de dicho organismo internacional no lograron, en la conferencia de Buenos Aires, consolidar unas 25 propuestas, incluyendo un llamado a establecer un foro central de negociación en e-commerce (Reuters, 2017). Queda, por tanto, esperar la siguiente conferencia ministerial, a efectos de poder dilucidar si es que los miembros adoptan una postura más radical y coherente con los cambios tecnológicos —incluyendo el crecimiento sostenido de internet— que a la fecha venimos experimentando. Es innegable, tal como lo sostiene Panagariya, que el comercio electrónico ofrece oportunidades sin precedentes tanto para los países en desarrollo como para los desarrollados (Panagariya, 2000, p. 978), y que estos deben ser aprovechados de manera equitativa, por todos los miembros de la OMC.

\section{Subvenciones a la pesca}

El siguiente acuerdo a ser analizado es la Decisión Ministerial sobre Subvenciones a la Pesca, la cual fuese adoptada el 13 de diciembre del 2017. Esta decisión reconoce el esfuerzo llevado a cabo por los Miembros en la reglamentación de esta disciplina, y hace además hincapié en la necesidad de adoptar, en la Conferencia Ministerial de 2019, «un acuerdo sobre disciplinas amplias y eficaces que prohíban ciertas formas de subvenciones a la pesca que contribuyen a la sobrecapacidad y la sobrepesca, y eliminen las subvenciones que contribuyen a la pesca INDNR» ${ }^{13}$. Esto pues, lleva a formularnos la pregunta: ¿qué constituyen las subvenciones a la pesca, y cuál es el motivo por el cual esta práctica genera preocupación al interior de la $\mathrm{OMC}$ ?

La creciente preocupación por las subvenciones a la pesca no es un tema nuevo en la agenda de la OMC, sino más bien constituye un reflejo de las discusiones sobre conservación y sostenibilidad de los recursos marinos, las cuales se remontan a la década de los noventa. A manera de ejemplo, en el marco de la Conferencia Internacional sobre la Pesca Responsable en Cancún (1992) se respaldó la preparación de un Código de Conducta para la Pesca Responsable (FAO, 2003, p. 1), mientras que, en ese mismo año, la Organización de las Naciones Unidas instó a «eliminar o reducir las subvenciones que no se ajusten a los objetivos del desarrollo sostenible» y a «reformar las estructuras existentes de incentivos económicos y fiscales para alcanzar los objetivos del medio ambiente y el desarrollo» (Vergaray, 2016).

\footnotetext{
12 Como ha podido ser apreciado, la inclusión del «e-commerce» dentro de la esfera de actuación del AGCS conlleva ciertos problemas que, a la fecha, continúan siendo materia de discusión.

13 Pesca ilegal, no declarada, y no reglamentada (INDNR). El texto completo de esta decisión puede ser encontrado en el siguiente documento OMC: WT/L/1031.
} 
Posterior a ello, en setiembre del 2015, más de 150 jefes de Estado y de Gobierno se reunieron en la Cumbre del Desarrollo Sostenible en la que aprobaron la Agenda 2030, la cual contiene 17 objetivos de aplicación universal que, desde el 1 de enero de 2016, rigen los esfuerzos de los países para lograr un mundo sostenible en el año 2030. Cabe destacar que estos objetivos «presentan la singularidad de instar a todos los países, ya sean ricos, pobres o de ingresos medianos, a adoptar medidas para promover la prosperidad» (ONU, s.f. a), y al mismo tiempo, proteger al planeta. En ese contexto:

En la meta 14,6 de los $\mathrm{ODS}^{14}$ se pide prohibir ciertas formas de subvenciones a la pesca que contribuyen a la sobrecapacidad y la pesca excesiva, eliminar las subvenciones que contribuyen a la pesca ilegal, no declarada y no reglamentada (pesca INDNR) y abstenerse de introducir nuevas subvenciones de esa índole de aquí a 2020. En la meta se reconoce asimismo que la negociación sobre las subvenciones a la pesca en el marco de la OMC debe incluir un trato especial y diferenciado, apropiado y efectivo para los países en desarrollo y los países menos adelantados. (OMC, 2017, p. 41).

Dicho esto, en el marco del sistema multilateral de comercio, los miembros de la OMC, en la Conferencia Ministerial de Doha, decidieron poner en marcha negociaciones para aclarar y mejorar las disciplinas relativas a las subvenciones a la pesca, siendo que en la Conferencia Ministerial de Hong Kong del 2005 hubo un amplio acuerdo en fortalecer esas disciplinas, incluso «mediante la prohibición de determinadas formas de subvenciones a la pesca que contribuyan al exceso de capacidad y la sobrepesca» (OMC, s.f. j). Ello, evidentemente, refleja una preocupación global en esta materia, ya que, de acuerdo con cifras recientes, al menos el $50 \%$ de las poblaciones de peces son explotadas completamente, y el 25\% están sobreexplotadas, agotadas o recuperándose del agotamiento (Young, 2009, p. 478), ello debido a la sobrepesca. Dicho escenario guarda estrecha relación con las subvenciones otorgadas en este sector, las cuales — según la FAO, de acuerdo con un estudio realizado en 1989- habrían alcanzado un valor de US\$ 22 billones (medidos en términos de costos operativos) y de 54 billones de dólares, incluyendo la totalidad de costos de inversión (Grynberg y Rochester, 2005, p. 505).

Dentro del régimen de la $\mathrm{OMC}$, las disciplinas relacionadas con la pesca se encuentran comprendidas en el Acuerdo sobre Subvenciones y Medidas Compensatorias (Acuerdo SMC) ${ }^{15}$ y no dentro del Acuerdo sobre Agricultura. Es, justamente aquí, donde descansan varias de las críticas a este tema, ya que, según diversos ambientalistas y miembros de la OMC, el citado instrumento es poco efectivo para abordar

\footnotetext{
14 Objetivos de Desarrollo Sostenible (nota a pie de página ańadida).

15 En ese contexto, una subvención puede ser definida como una contribución financiera de un gobierno o de cualquier organismo público en el territorio de un Miembro, la cual otorga un beneficio.
} 
problemas específicos relacionados con la sobrepesca y con sus potenciales efectos de distorsión al comercio; ello, debido a que — según señalan - el sector pesquero es único en varios aspectos (Chang, 2003, p. 880).

De acuerdo con la OMC, existen cuatro grandes categorías de subvenciones pesqueras: a) subvenciones al sector de las capturas; b) subvenciones a la industria de la construcción naval; c) subvenciones a la industria elaboradora; d) otras subvenciones diversas, en particular las relacionadas con la investigación y el desarrollo o la comercialización (OMC, s.f. k, párr. 31). Esta lista — según lo señalado por el citado organismo - no es exhaustiva, siendo que la misma habría sido realizada sobre la base de las subvenciones notificadas por los Miembros desde el 1 de enero de 1995.

A efectos de clarificar dicho panorama, las subvenciones en cuestión habrían revestido la forma de, por ejemplo, ventajas fiscales (a través de un 50 por ciento de descuento en el impuesto sobre las compras o la propiedad) concedidas a «las personas que construyan o importen» buques, entre ellos, «buques pesqueros de navegación marítima» (OMC, s.f. l); subvenciones concedidas a organizaciones de ventas encargadas de distribuir «ayuda para transporte otorgada al sector pesquero"; subvenciones concedidas a organizaciones de ventas a cargo de distribuir «ayuda a determinados sectores de la industria pesquera", y además, mediante ayudas concedidas a "grupos de compañías que producen o venden pescado o productos de pescado noruegos [...]» (OMC, s.f. m); entre otras.

Partiendo de lo antes señalado, queda claro que el objeto de las negociaciones es poder adoptar, en la siguiente Conferencia Ministerial del 2019, un acuerdo sobre las subvenciones a la pesca que dé cumplimiento al ODS 14.6; es decir, un acuerdo que prohíba aquellas subvenciones que contribuyen a la pesca excesiva y sobrecapacidad, y que elimine otras que fomentan la pesca INDNR. Si bien el trabajo no ha resultado sencillo -recuérdese que las negociaciones sobre este tema se han extendido por más de una década- lo cierto es que el compromiso en poder alcanzar las metas trazadas es innegable: tan es así que los Miembros de la OMC, reunidos el 30 de enero del 2018 en el marco del Grupo de Negociación sobre las Normas, examinaron la forma de organizar sus trabajos sobre las subvenciones a la pesca, con el fin de dar cumplimiento al objetivo planteado (OMC, s.f. n).

\section{Derechos de propiedad intelectual}

La tercera y última decisión ministerial a ser analizada tiene que ver con la labor realizada por el Consejo de los Aspectos de los Derechos de Propiedad Intelectual relacionados con el Comercio, relativa a las reclamaciones no basadas en una infracción 
y las reclamaciones en casos en que existe otra situación en el ámbito de los ADPIC. En ese contexto, se acordó continuar con el examen del alcance y las modalidades de las reclamaciones de los tipos previstos en los apartados b) y c) del párrafo 1 del artículo XXIII del GATT de 1994, y no imponer tales reclamaciones en el ámbito del Acuerdo sobre los ADPIC, mientras dure el citado examen. ${ }^{16}$

\subsection{Sobre el ADPIC}

El Acuerdo de la OMC sobre los ADPIC — negociado en la Ronda Uruguay— establece niveles mínimos de protección que cada gobierno ha de otorgar a la propiedad intelectual de los demás Miembros de la OMC. En ese contexto, el acuerdo antes citado:

[...] define cada uno de los principales elementos de la protección: la materia que ha de protegerse, los derechos que han de conferirse y las excepciones permisibles a esos derechos, y la duración mínima de la protección. El Acuerdo establece esas normas exigiendo, en primer lugar, que se cumplan las obligaciones sustantivas estipuladas en los principales convenios de la OMPI: el Convenio de París para la Protección de la Propiedad Industrial (el Convenio de París) y el Convenio de Berna para la Protección de las Obras Literarias y Artísticas (el Convenio de Berna) en sus versiones más recientes. Con excepción de las disposiciones del Convenio de Berna sobre los derechos morales, todas las principales disposiciones sustantivas de esos Convenios se incorporan por referencia al Acuerdo sobre los ADPIC y se convierten así en obligaciones para los países Miembros de dicho Acuerdo. (OMC, s.f. o).

Es importante mencionar que el Acuerdo bajo análisis incorpora obligaciones adicionales en aspectos que los convenios antes existentes no tratan o tratan de modo que se consideró insuficiente. Además, el instrumento en cuestión recoge algunos principios generales aplicables a todos los procedimientos de observancia de los derechos de propiedad intelectual, y contiene disposiciones sobre procedimientos y recursos civiles, penales y administrativos, medidas provisionales, y disposiciones especiales relacionadas con las medidas en frontera (OMC, s.f. o). Finalmente, debe mencionarse que, al igual que el GATT y el AGCS, el ADPIC recoge también los principios de no discriminación (cláusula de nación más favorecida y trato nacional), y reconoce también la posibilidad de acudir al procedimiento de solución de diferencias de la OMC, en caso de controversias respecto al cumplimiento de las obligaciones previstas.

16 El texto completo de esta decisión se encuentra disponible en el siguiente documento OMC: WT/L/1033. 


\subsection{Sobre el artículo XXIII del GATT 1994}

En términos generales, el artículo XXIII del GATT 1994 faculta a los miembros a interponer reclamaciones a causa de la «anulación o menoscabo» de las ventajas que les corresponderían en virtud de dicho acuerdo, independientemente de la compatibilidad o incompatibilidad del GATT con las medidas en cuestión (Lacarte y Piérola, 2004, p. 16). En concreto, el párrafo 1 del citado artículo XXIII establece las condiciones en las cuales un Miembro puede recurrir al sistema de solución de diferencias. Este párrafo señala:

1. En caso de que una parte contratante considere que una ventaja resultante para ella directa o indirectamente del presente Acuerdo se halle anulada o menoscabada o que el cumplimiento de uno de los objetivos del Acuerdo se halle comprometido a consecuencia de:

a) que otra parte contratante no cumpla con las obligaciones contraídas en virtud del presente Acuerdo; o

b) que otra parte contratante aplique una medida, contraria o no a las disposiciones del presente Acuerdo; o

c) que exista otra situación, dicha parte contratante podrá, con objeto de llegar a un arreglo satisfactorio de la cuestión, formular representaciones o proposiciones por escrito a la otra u otras partes contratantes que, a su juicio, estime interesadas en ella. Toda parte contratante cuya intervención se solicite de este modo examinará con comprensión las representaciones o proposiciones que le hayan sido formuladas.

Como puede apreciarse, este párrafo prevé tres posibilidades u opciones para el reclamante en caso concurra anulación o menoscabo (OMC, s.f. p):

- La primera de ellas está referida a los casos en los cuales existe infracción: estas reclamaciones tienen lugar cuando se ha producido una anulación o menoscabo de una ventaja como consecuencia de que otro miembro de la OMC no cumpla con sus obligaciones contraídas en virtud del GATT de 1994.

- El segundo tipo de reclamaciones es el llamado «reclamaciones en los casos en que no existe infracción»: pueden presentarse para impugnar cualquier medida aplicada por otro Miembro, aunque no entre en conflicto con el GATT de 1994, siempre y cuando dé lugar a una "anulación o menoscabo de una ventaja» ${ }^{17}$.

- Finalmente, el tercer tipo de reclamaciones es el llamado «reclamaciones en casos en que existe otra situación»: estas reclamaciones podrían abarcar cualquier situación, siempre y cuando dé lugar a una «anulación o menoscabo». No obstante, si bien con el GATT 1947 se presentaron algunas reclamaciones de este tipo, ninguna de ellas dio lugar a un informe de un grupo especial. En la OMC, hasta

17 Como bien señala este organismo, ha habido pocas reclamaciones de este tipo, tanto en el marco del GATT de 1947 como dentro de la OMC. 
la fecha ningún reclamante ha invocado el párrafo 1 c) del artículo XXIII del GATT de 1994.

\subsection{Naturaleza del problema}

Tal como fuese mencionado con anterioridad, en virtud de la Decisión Ministerial del 13 de diciembre del 2017, los miembros acordaron continuar con el examen del alcance y las modalidades de las reclamaciones de los tipos previstos en los apartados b) y c) del párrafo 1 del artículo XXIII del GATT de 1994 (es decir, reclamaciones en casos en los cuales no exista infracción, y reclamaciones en casos en que exista «otra situación»), y no imponer tales reclamaciones en el ámbito del Acuerdo sobre los ADPIC, mientras dure el citado examen.

Uno de los problemas que puede apreciarse radica en que, de permitirse la interposición de reclamos de esta naturaleza, existiría el riesgo de alcanzar resultados incoherentes en los eventuales procedimientos a ser tramitados en el marco del GATT, AGCS y ADPIC (no debe olvidarse que los derechos de propiedad intelectual están presentes en todos los casos relacionados con bienes o servicios) (OMC, s.f. q). Según una comunicación cursada por el Canadá, la República Checa, las entonces Comunidades Europeas, Hungría y Turquía, ello resultaría particularmente relevante en los siguientes escenarios:

Un país puede optar por prohibir la fabricación, elaboración, importación y distribución de materiales o productos que contienen una sustancia prohibida, lo que podría afectar a titulares extranjeros de derechos de propiedad intelectual que son los principales suministradores de esos productos al mercado. De forma análoga, otro país puede decidir imponer restricciones a la publicidad en la comercialización de ciertos bienes o servicios, lo que podría entrańar importantes limitaciones a la promoción de marcas, marcas registradas de fábrica o de comercio u otras denominaciones. Por último, un país puede optar por imponer una pesada carga impositiva a ciertos bienes con derechos de propiedad intelectual cuya utilización es sumamente perjudicial para el medio ambiente. (OMC, s.f. q).

De manera adicional, según lo señalado en esta misma comunicación:

En todos estos casos es muy dudoso que los Miembros de la OMC se demuestren inclinados a dar a los países la opción de presentar una reclamación sin que exista infracción de disposiciones en el marco del Acuerdo sobre los ADPIC, en caso de que se constate que la medida cumple plenamente las disposiciones multilaterales del GATT y sus acuerdos anexos o del AGCS. Como mínimo, a fin de mantener la coherencia entre los Acuerdos de la OMC, las medidas que estén amparadas por las excepciones generales establecidas en el GATT o en el AGCS no deberían quedar sujetas al proceso de solución de diferencias en el marco del Acuerdo sobre los ADPIC. De 
los acuerdos enumerados en la nota de la Secretaría ${ }^{18}$ que contienen disposiciones sobre las reclamaciones en casos en que no hay infracción se deduce que cabe reducir la posibilidad de conflictos de esa naturaleza. Además de las excepciones hay también otros enfoques, que valdría la pena examinar en posteriores debates. (OMC, s.f. q).

Un problema adicional, según lo señalado por Stilwell y Tuerk, radica en que la existencia de reclamaciones sin infracción podría conllevar a un aumento en el nivel de protección impuesto por determinados países, más allá de los requisitos mínimos establecidos en el ADPIC, o a un escenario en el que estos países se abstengan de utilizar medidas compatibles con el citado acuerdo; como, por ejemplo, licencias obligatorias. En este contexto, las reclamaciones de este tipo podrían utilizarse como un mecanismo de presión para países en desarrollo, para que no exploren plenamente su derecho a utilizar dichas licencias, a efectos de poder garantizar el acceso a medicamentos esenciales (Stilwell y Tuerk, 2001, p. 7).

Finalmente, tal como señalan estos autores, existen precedentes limitados, tanto por parte de grupos especiales como del Órgano de Apelación de la OMC, sobre cómo podrían aplicarse reclamaciones sin infracción en el marco del ADPIC. De hecho, solo tres reclamaciones de esta naturaleza fueron exitosas en toda la historia del GATT, lo cual evidentemente deja a los miembros de la OMC con pocas herramientas sobre cómo abordar este tema (recuérdese que en el ADPIC fue adoptado en el marco de la OMC y no durante el GATT 1947).

En conclusión; existen varias razones por las cuales los miembros han decidido no imponer reclamaciones en casos en los cuales no exista infracción, y reclamaciones en casos en que exista "otra situación" en el marco del ADPIC, ello desde la entrada en vigencia del citado acuerdo. Sea cual sea el resultado del examen que los miembros llevan efectuando a la fecha, lo cierto es que, desde nuestro punto de vista, es poco probable que reclamos de este tipo puedan ser autorizados, dentro del régimen multilateral de comercio.

\section{Conclusiones}

La Conferencia Ministerial celebrada en Buenos Aires ha dejado como resultado la adopción de diversas decisiones ministeriales, con compromisos a ser asumidos por todos los miembros de la OMC. Si bien muchos de los temas que motivaron la emisión de estas decisiones están siendo materia de discusión desde hace muchos años, lo cierto es que la adopción de compromisos por consenso es una tarea difícil,

18 Documento IP/C/W/124, de fecha 28 de enero de 1999. 
dado que ello amerita una evaluación cuidadosa de las ventajas y desventajas que ello puede acarrear a cada país negociador.

Sin perjuicio de las dificultades inherentes a todo proceso de negociación, lo cierto es que la Ronda de Doha lleva más de una década y media de iniciada, sin prospecto alguno de que pueda ser finalizada, al menos en el corto plazo. Partiendo de ello, urge la adopción de decisiones que puedan encaminarla a buen término, no solo para evitar el socavamiento de la legitimidad del sistema multilateral de comercio, sino también para fortalecer la imagen de la OMC en temas tan sensibles como lo pueden ser, por ejemplo, el cuidado del medio ambiente (ello, a través de una decisión que impida el mantenimiento de ciertas subvenciones pesqueras, que contribuyen a la pérdida de recursos marinos). Para ello, es necesaria la adopción de compromisos efectivos en los siguientes aspectos:

- Clasificación del comercio electrónico dentro del marco del AGCS, y las eventuales consecuencias derivadas de considerar dicha disciplina dentro de los módulos 1 y 2 del citado acuerdo.

- Subvenciones a la pesca, de manera tal que el acuerdo a ser adoptado dé cumplimiento al ODS 14.6, a través de la prohibición de aquellas subvenciones que contribuyen a la pesca excesiva y sobrecapacidad, y la eliminación de otras que fomentan la pesca INDNR.

- Reclamaciones no basadas en una infracción y las reclamaciones en casos en que existe otra situación en el ámbito de los ADPIC. La adopción de decisiones en este ámbito deberá tener en cuenta las preocupaciones planteadas por diversos miembros, tal como han sido descritas a lo largo del presente estudio.

Queda por esperar cuáles serán los resultados de la próxima conferencia ministerial, a ser celebrada el año próximo, y los compromisos que cada miembro de este organismo internacional está dispuesto a asumir, no solo para el beneficio de la OMC, sino también para la comunidad internacional.

\section{Referencias}

Chang, S.W. (2003). WTO disciplines on fisheries subsidies: A historic step towards sustainability? Journal of International Economic Law, 6(4), 879-921. https://doi. org/10.1093/jiel/6.4.879

FAO. (2003). Technical Guidelines for Responsible Fisheries: Fisheries Management. 2. The Ecosystem Approach to Fisheries. Roma: FAO. Recuperado de http://www.fao.org/3/ay4470e.pdf 
Grynberg, R. y Rochester, N. (2005). The emerging architecture of a World Trade Organization Fisheries Subsidies Agreement and the interests of developing coastal states. Journal of World Trade, 39(3), 503-526.

Lacarte, J. y Piérola F. (2004). Estudio comparativo de los mecanismos de solución de diferencias del GATT y de la OMC: ¿Qué se logró en la Ronda Uruguay? En J. Lacarte y J. Granados (Eds.). Solución de controversias comerciales inter-gubernamentales: Enfoques multilaterales y regionales (pp. 13-32). Buenos Aires: Banco Interamericano de Desarrollo.

Mitchell, A.D. (2001). Towards compatibility: the future of electronic commerce within the global trading system. Journal of International Economic Law, 4(4), 683-723. https:// doi.org/10.1093/jiel/4.4.683

OMC (s.f. a). Miembros y Observadores. Recuperado de https://www.wto.org/spanish/ thewto_s/whatis_s/tif_s/org6_s.htm

OMC (s.f. b). Las Conferencias Ministeriales. Recuperado de https://www.wto.org/spanish/ thewto_s/minist_s/minist_s.htm

OMC (s.f. c). Finaliza la conferencia ministerial con decisiones sobre las subvenciones a la pesca y los derechos sobre el comercio electrónico; la labor en curso continúa. Recuperado de https://www.wto.org/spanish/news_s/news17_s/mc11_13dec17_s.htm

OMC (s.f. d). Programa de Trabajo sobre el Comercio Electrónico. Adoptado por el Consejo General el 25 de setiembre de 1998. Documento WT/L/274, de fecha 30 de setiembre de 1998, párrafo 1.3.

OMC (s.f. e). Consecuencias del comercio electrónico para el desarrollo. Nota de la Secretaría. Documento WT/COMTD/W/51, de fecha 23 de noviembre de 1998, párrafo 3.

OMC (s.f. f). Comercio Electrónico. Recuperado de https://www.wto.org/spanish/tratop_s/ ecom_s/ecom_s.htm

OMC (s.f. g). Debate Especifico sobre el Comercio Electrónico celebrado el 15 de junio de 2001 bajo los auspicios del Consejo General. Resumen de las cuestiones planteadas preparado por la Secretaría. Documento WT/GC/W/436, de 6 de julio del 2001.

OMC (s.f. h). Módulo de formación sobre el AGCS: Capitulo 1 - Finalidad y conceptos básicos. Recuperado de https://www.wto.org/spanish/tratop_s/serv_s/cbt_course_s/c1s3p1_s. htm

OMC (s.f. i). WTO E-Campus. Módulo 6. Estructura modal del AGCS. Recuperado de https:// ecampus.wto.org/admin/files/Course_413/Module_970/ModuleDocuments/ GATS_M6_R1_S.pdf

OMC (s.f. j). Nota Informativa: Negociaciones sobre las normas: disciplinas antidumping y sobre subvenciones (incluidas las subvenciones a la pesca) y acuerdos comerciales regionales. Recuperado de https://www.wto.org/spanish/thewto_s/minist_s/mc10_s/briefing_ notes_s/brief_antidumping_s.htm

OMC (s.f. k). Normas del GATT/OMC sobre subvenciones y ayudas otorgadas en la industria pesquera. Nota de la Secretaría. Documento WT/CTE/W/80, de 9 de marzo de 1998, párrafo 31 .

OMC (s.f. 1). Documentos G/SCM/N/25/KOR y G/SCM/N/3/KOR/Rev.1. 
OMC (s.f. m). Documentos G/SCM/N/3/NOR-L/7611/Add.14; G/SCM/N/16/NOR; y G/SCM/N/25/NOR

OMC (s.f. n). Negociaciones sobre las subvenciones a la pesca. Los Miembros de la OMC examinan la forma de organizar sus trabajos en el marco de las negociaciones sobre las subvenciones a la pesca después de la Undécima Conferencia Ministerial. Recuperado de https://www.wto.org/spanish/news_s/news18_s/fish_30jan18_s.htm

OMC (s.f. o). Acuerdo sobre los ADPIC: visión general. Recuperado de https://www.wto.org/ spanish/tratop_s/trips_s/intel2_s.htm

OMC (s.f. p). Módulo de Formación sobre el Sistema de Solución de Diferencias: Capitulo 4. Fundamento jurídico de una diferencia. Recuperado de https://www.wto.org/spanish/ tratop_s/dispu_s/disp_settlement_cbt_s/c4s2p1_s.htm

OMC (s.f. q). Reclamaciones en casos en que no hay infracción, de conformidad con el acuerdo sobre los ADPIC - Temas propuestos para el examen del alcance y las modalidades previsto en el párrafo 3 del artículo 64 del acuerdo sobre los ADPIC. Comunicación del Canadá, la República Checa, las Comunidades Europeas y sus Estados miembros, Hungría y Turquía. Documento IP/C/W/191, de 22 de junio del 2000, párrafo 8.

OMC. (2017). Informe Anual 2017. Recuperado de https://www.wto.org/spanish/news_s/ news17_s/anrp_31may17_s.htm, p. 41.

Panagariya, A. (2000). E-commerce, WTO and developing countries. The World Economy, 23(8), 959-978.

Some WTO Members to push for e-commerce rules as broader deal fails (13 de diciembre del 2017). Reuters. Recuperado de https://www.reuters.com/article/us-trade-wto-digital/ some-wto-members-to-push-for-e-commerce-rules-as-broader-deal-fails-idUSKBN1E72YV

Stilwell, M. y Tuerk, E. (2001). Non-violation complaints and the TRIPS Agreement: Some considerations for WTO Members. Center for International Environmental Law, p. 7.

ONU - Organización de Naciones Unidas (s.f. a). Objetivos de Desarrollo Sostenible. 17 objetivos para transformar nuestro mundo. Recuperado de http://www.un.org/ sustainabledevelopment/es/la-agenda-de-desarrollo-sostenible/

Vergaray, M.d.C (18 de octubre del 2016). Subvenciones a la pesca: estado actual y perspectivas para su respectiva regulación internacional. International Centre for Trade and Sustainable Development (ICTSD). Recuperado de https://www.ictsd.org

Young, M.A. (2009). Fragmentation or Interaction: the WTO, fisheries subsidies, and international law. World Trade Review, 8(4), 477-515. https://doi.org/10.1017/ s1474745609990140

Fecha de recepción: 15 de marzo de 2018 Fecha de aprobación: 25 de junio de 2018 